Total CSF and serum $A D A$ and $C S F A D A_{2}$ in patients with meningitis or a normal lumbar puncture (LP)

\begin{tabular}{lllllll}
\hline Diagnosis & Total ADA & CSF $A D A_{2}$ & $\%$ \% ADA & \multicolumn{2}{l}{ Serum Total ADA } \\
\hline TBM (n=11) & $6.9(4.2-69)$ & $7.0(3.5-57)$ & 88.1 & $(80.5-100)$ & 47.8 & $(43.5-85.1)$ \\
CM (n=9) & $4.4(2.5-10.9)$ & $3.9(1.2-6.1)$ & $68.2^{\star}$ & $(43.6-93.2)$ & 45.9 & $(26.6-48.7)$ \\
ABM (n=13) & $3.6(1.1-11.4)$ & $1.7(0.1-5.4)$ & 37.9 & $(5.6-56.7)$ & 15.7 & $(7.0-34.4)$ \\
Aseptic (n=9) & $0.3(0-2.7)$ & - & - & & 25.7 & $(10.3-42.4)$ \\
Normal LP (n=19) & $0(0-3.7)$ & - & - & & 37.3 & $(10.7-97.8)$ \\
p Value (ANOVA) & 0.005 & 0.027 & $<0.0001$ & \multicolumn{2}{c}{$<0.0001$} \\
\hline
\end{tabular}

All results are median and range, except for $\% \mathrm{ADA}_{2}$ (mean and range). TBM=tuberculous meningitis; $\mathrm{CM}=$ cryptococcal meningitis; $\mathrm{ABM}=$ acute bacterial meningitis.

*Three subjects with $\mathrm{CM}$ had $\% \mathrm{ADA}_{2}>80 \%$.

Data were analysed using EpiInfo 6.04 (CDC, Atlanta) and PRISM 2.01 (GraphPad Software, USA). Continuous variables were compared using analysis of variance (ANOVA) and a $5 \%$ level of significance was used.

Comparison of total CSF and serum ADA and CSF $\mathrm{ADA}_{2}$ in the diagnostic categories is shown in the table. Total CSF ADA was highest in patients with tuberculous meningitis. Using a cut off of $\geqslant 6 \mathrm{U} / 1$, the test was $90.9 \%$ sensitive in detecting tuberculous meningitis (10 of 11). The specificity was $94 \%$ (47 of 50 ) in all patients and $77.3 \%$ (17 of 22) compared with those with cryptococcal meningitis or acute bacterial meningitis. There were no significant differences between those with tuberculous meningitis established by culture and probable disease Similarly, there were no significant differences in the CSF ADA concentrations in HIV positive and negative patients in the acute bacterial meningitis (mean $4.88 \mathrm{U} / 1$ vs 3.71 $\mathrm{U} / 1 ; \mathrm{p}=0.49$ ) and normal lumbar puncture groups (mean $0.74 \mathrm{U} / 1 \cup 0.12 \mathrm{U} / \mathrm{l} ; \mathrm{p}=0.14$ ). Serum ADA concentrations were highest in patients with tuberculous meningitis or cryptococcal meningitis and significantly lower in those with acute bacterial meningitis.

There were significant differences in the mean proportion of total ADA that comprised $\mathrm{ADA}_{2}$ in patients with tuberculous meningitis, cryptococcal meningitis, or acute bacterial meningitis $(p<0.001)$. Using a cut off of $80 \%$ for the proportion of CSF $\mathrm{ADA}_{2}$, the test was $100 \%$ sensitive and $86.4 \%$ specific in detecting tuberculous meningitis (positive predictive value $78.6 \%$, negative predictive value $100 \%$ ). An $\mathrm{ADA}_{2}$ of $>80 \%$ was found in three of nine of those with cryptococcal meningitis and none with acute bacterial meningitis. A cut off of $90 \%$ changed the sensitivity and specificity to $36.4 \%$ (four of 11 ) and $95.5 \%$ (21 of 22) respectively.

The diagnosis of tuberculous meningitis remains a challenge. Acid fast bacilli are typically identified by microscopy in less than a quarter of patients and mycobacterial culture, the present "gold standard" for diagnosis is positive in $45 \%-90 \%$ of cases and may take up to 8 weeks to yield results. Recent advances in rapid automated liquid culture may reduce culture times. The use of the polymerase chain reaction (PCR) to detect Mycobacterium tuberculosis specific DNA may be of potential value; however, problems with its specificity have been encountered.

Sensitivities of $100 \%$ and specificities of $99 \%{ }^{3}$ have been described for total CSF ADA. However, others have reported lower sensitivities and specificities and have suggested that CSF ADA measurement has "no advantages over conventional diagnostic criteria". Possible reasons for the varying usefulness in different studies include different disease profiles, times to presentation, and ages, as children with tuberculous meningitis have lower ADA values. ${ }^{4}$
We were able to differentiate patients with tuberculous meningitis from those with aseptic meningitis or a normal lumbar puncture on the basis of the total CSF ADA. However, there was overlap between patients with tuberculous meningitis and those with cryptococcal meningitis or acute bacterial meningitis. A proportion of $\mathrm{ADA}_{2}$ isoenzyme of $>80 \%$ seems to be a reliable marker of tuberculous meningitis, yielding a sensitivity of $100 \%$ and specificity of $86.4 \%$. The only other diagnostic category with patients who had $>80 \% \mathrm{ADA}_{2}$ was cryptococcal meningitis, which is easily diagnosed on indian ink staining and serology. Serum ADA concentrations were not useful in differentiating the cause of meningitis.

The laboratory technique for measuring $\mathrm{ADA}_{2}$ is inexpensive (about $£ 1$ per test), relatively simple to perform, and can be adapted to an autoanalyser. It may thus be used in laboratories with limited resources. Measurement of $\mathrm{ADA}_{2}$ produces results rapidly, thus potentially decreasing delays before therapy for tuberculous meningitis is initiated. These results seem promising and may make a valuable contribution to the early and accurate diagnosis of tuberculous meningitis. The use of CSF ADA, in the diagnosis of tuberculous meningitis should be further evaluated in larger series, including patients with other lymphocytic meningitides and different settings.

We thank the study patients; also Sally Ann Martin, Mark Ferreira, Renee Wilson, and laboratory staff a Gold Fields West Hospital; Janice Paiker and staff of the department of Chemical Pathology, South African Institute for Medical Research (SAIMR). PS was funded by the Epidemiology Research Unit, Department of Health and Gold Fields of South Department of Health and Gold Fields of South Africa. ES is supported by the Special Trustees of St
Thomas' Hospital.

S EINTRACHT

Department of Chemical Pathology, South African Institute for Medical Research and University of the Witwatersrand, Johannesburg, South Africa

E SILBER

Department of Neurology, Baragwanath Hospital and University of the Witwatersrand; and Department of Clinical Neurosciences, Guy's, King's and St Thomas' School of Medicine, King's College, London, UK P SONNENBERG

Department of Community Health, University of the Witwatersrand; and Infectious Disease Epidemiology Unit, London School of Hygiene and Tropical Medicine, London, UK

H J KOORNHOF

Department of Medical Microbiology, South African Institute for Medical Research and University of the Witwatersrand

D SAFFER

Department of Neurology, Baragwanath Hospital and University of the Witwatersand

Correspondence to: Dr E Silber, Department of Neuroimmunology, 2nd Floor, Hodgkin Building, Guy's Hospital, London, SE1 9RT,

UKeli.silber@kcl.ac.uk
1 Ungerer JP, Oosthuizen HM, Bissbort SH, et al. Serum adenosine deaminase: isoenzymes and diagnostic application. Clin Chem 1992;38: $1322-6$

2 Gakis C. Adenosine deaminase (ADA) isoenzymes ADA1 and ADA2: diagnostic and biological role. Eur Respir F 1996;9:632-3.

3 Ribera E, Martinez-Vazquez JM, Ocana I, et al. Activity of adenosine deaminase in cerebrospinal fluid for the diagnosis and follow-up of tuberculous meningitis in adults. F Infect Dis 1987;155:603-7.

4 Donald P, Malan C, van der Walt A, et al. The simultaneous determination of cerebrospinal fluid and plasma adenosine deaminase activity as a diagnostic aid in tuberculous meningitis. $S$ as a diagnostic aid in tubercul
Afr Med $f 1986 ; 69: 505-7$

5 Silber E, Sonnenberg P, Ho KC, et al. Meningitis in a community with a high prevalence of tuberculosis and HIV infection. If Neurol Sciences 1999;162:20-7.

CORRESPONDENCE

\section{Unilateral focal lesions in the rostrolateral medulla influence chemosensitivity and breathing measured during wakefulness, sleep, and exercise}

We have read with interest the article entitled Unilateral focal lesions in the rostrolateral medulla influence chemosensitivity and breathing measured during wakefulness, sleep, and exercise by Morrell et al, ${ }^{1}$ which shows that unilateral ischaemic lesions of the rostrolateral medulla may lead to an abnormal ventilatory $\mathrm{CO}_{2}$ response and sleep apnoea. We have recently conducted a similar study on five patients with syringobulbia. Syringobulbia has a predilection for autonomic nuclei of the cardiorespiratory network localised in the caudal medulla and this may cause severe respiratory and cardiovascular abnormalities. ${ }^{2}$ On occasion, a syrinx may extend to the rostral and ventral medulla. ${ }^{3}$

Out of five patients with syringobulbia studied with MRI, ventilatory $\mathrm{CO}_{2}$ response and polysomnography, one showed bilateral syringomyelic cavities in the caudal dorsal medulla with unilateral extension to the rostrolateral medulla (figure). This 40 year old patient showed the following respiratory abnormalities: end tidal $\mathrm{CO}_{2}, 47.3 \mathrm{~mm} \mathrm{Hg}$; p $0.1,0.21$; ventilatory $\mathrm{CO}_{2}$ response, 1.78 $1 / \mathrm{mm} \mathrm{Hg}$; apnoea index, 52 events/hour of sleep, with a total number of 212 obstructive sleep apnoeas, four central apnoeas, and 39 hypopnoeas. Maximal duration of obstructive sleep apnoeas was 125 seconds and oxygen saturation values during apnoeic episodes lower than $50 \%$. There was also evidence of severe autonomic dysfunction with orthostatic hypotension, arterial hypotension at rest, and complete loss of sinus arrhythmia. Despite the severity of the respiratory abnormalities recorded, the patient refused to receive any respiratory support, and to date has not developed any cardiorespiratory complication during a follow up of 9 years.

It seems that extension of the syrinx to ventral and rostral medullary areas may lead to more severe respiratory and cardiovascular abnormalities. Three stages in the progression of syringobulbia may be described in involvement of autonomic and respiratory structures: (1) initial involvement of the 


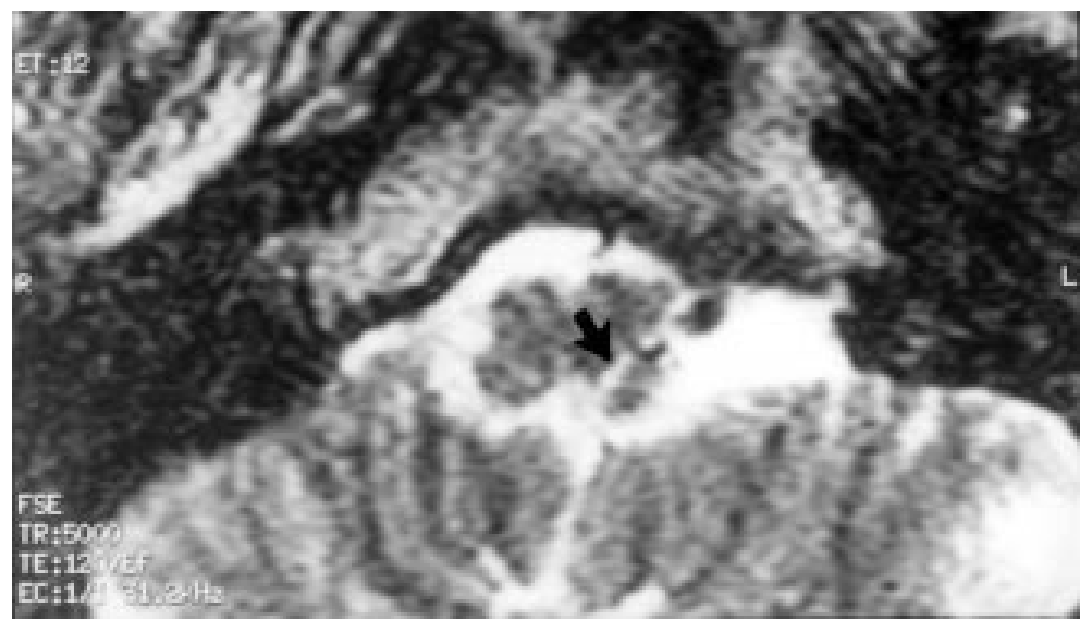

T2 weighted axial MRI through the upper medulla showing a small hyperintensive linear lesion running from the dorsal surface to the ventrolateral surface of the medulla.

caudal and dorsolateral medulla with damage to the nucleus tractus solitarius, vagal motor nucleus, and nucleus ambiguus which may impair cardiovascular reflexes; (2) ventral extension with involvement of the intermediate reticular formation; and (3) further ventral and rostral extension to the anterolateral surface of the rostral medulla, involving vasomotor neurons and central chemoreceptors. These last two stages may be accompanied by severe respiratory abnormalities and arterial hypotension.

We have encountered similar difficulties to those described by Morrell et $a l^{1}$ in outlining small lesions in the medulla by using MRI. Unless the cervicomedullary junction is studied with thin slices, small cavities may be easily overlooked. In such patients, chest and abdominal movements due to respiratory difficulties contribute to poor MRI definition.

MARTÍN NOGUÉS

Instituto de Investigaciones Neurológicas Dr Raúl Carrea (FLENI), Montañeses 2325, (1428) Buenos Aires, Argentina

KIRSTEN HEIDEL EDUARDO BENARROCH Department of Neurology, Mayo Clinic, Rochester, $M N, U S A$

Correspondence to: Dr Martín Nogués mnogues@fleni.org.ar

1 Morrell MJ, Heywood P, Moosavi SH, et al. Unilateral focal lesions in the rostrolatera medulla influence chemosensitivity and breathing measured during wakefulness, sleep, and exercise. I Neurol Neurosurg Psychiatry 1999;67:637-45

2 Nogués M, Gené R, Benarroch E, et al. Respiratory disturbances during sleep in syringomyelia and syringobulbia. Neurology 1999;52:177783.

3 Jonesco-Sisesti N. Syringobulbia. A contribution to the pathophysiology of the brainstem. New York: Praeguer, 1932.

The authors reply:

We were interested to read the paper by Nogues et $a l,{ }^{1}$ and we are grateful to the authors for bringing it to our attention. One of the aims of our study was to investigate the effect of unilateral lesions in humans at sites previously defined as being important for chemosensitivity in animals. For this reason we studied patients with relativity small focal lesions in the rostrolateral medulla. ${ }^{2}$ Nogues et al have investigated the effect of syringomyelia and syringobulbia on chemosensitivity and breathing during sleep; the MR images of their patients show the lesions to be more extensive than in our study. Nevertheless, both papers highlight the gross sleep apnoea in these patients and the importance of carrying out nocturnal polysomnography to identify any abnormalities in breathing during sleep; this point is emphasised in an excellent editorial by Malow. ${ }^{3}$

M J MORRELL A GUZ

National Heart and Lung Institute, Imperial College School of Medicine, Charing Cross Campus, St Dunstan's Road, London W6 8RP

1 Nogues M, Gene R, Benarroch E, et al. Respiratory disturbances during sleep in syringomyelia
and syringobulbia. Neurology 1999;52:177783.

2 Morrell MJ, Heywood P, Moosavi SH, et al. Unilateral focal lesions in the rostrolateral medulla influence chemosensitivity and breathing measured during wakefulness, sleep and exercise. $\mathcal{F}$ Neurol Neurosurg Psychiatry 1999;67: 637-45.

3 Malow BA. Wake up to the importance of sleep in neurological disorders. Neurology 1999;52: 1732-3.

\section{CORRECTION}

Davies NP, Eunson LH, Gregory RP, et al. Clinical, electrophysiological, and molecular genetic studies in a new family with paramyotonia congenita. $\mathcal{F}$ Neurol Neurosurg Psychiatry 2000;68:504-507. During the editorial process, figure 1 was reproduced incorrectly. The correct figure is shown here:

\section{BOOK REVIEWS}

Neurologic Catastrophes in the Emergency Department. By EeLCO FM wijdicks. (Pp266, £42.50). Published by Butterworth Heinemann, Oxford, 1999. ISBN 0-7506-7055-X.

One is naturally a little weary when asked to review a book entitled Neurologic Catastrophes in the Emergency Department. Why me? springs to mind. This said, swallowing my pride, I thoroughly enjoyed this book. Although the content is predictable enough; ischaemic stroke, haemorrhage, cord compression, infections etc, the presentation is special. It is an extremely accessible book, clearly laid out with key points of management and pharmacology appearing in shaded boxes (called capsules). The book is generously illustrated with informative diagrams (and I refer the reader, in particular, to that explaining assessment of the Glasgow coma scale) depicting procedures and anatomy. There are bountiful radiological images and a few select colour plates of clinical cases.

The text is a monograph but Dr Wijdick's opinions and practices are clearly stated as such and on the whole the evidence, or lack of it, for a management decision is described and referenced. Although American, the principles are transatlantic and this should in no way deter the English reader.

The content covers that dealt with by the neurologist, neurosurgeon, and casualty officer. It should be compulsory reading for those on the front line receiving such emergencies and may then play a part in preventing an emergency becoming a catastrophe. On the other hand, while providing the experienced neurologist with an enjoyable read, hopefully most are already fully acquainted with the up to date and appropriate management of a neurological emergency!

GILLIAN HALL

Handbook of Clinical Neurology; Systemic Diseases, Part III. By MJ AMINOFF and CG GOETZ (Handbook of Clinical Neurology series edited by PJ Vinken and GW Bruyn). (Pp748, US $\$ 264.50)$. Published by Elsevier Science, Amsterdam, 1998. ISBN 0444812903.

This is volume 71 in the series of the Handbook of Clinical Neurology. It is part III, the third and last volume dedicated to the neurology of systemic disease and, therefore, updates the previous volumes on this subject,

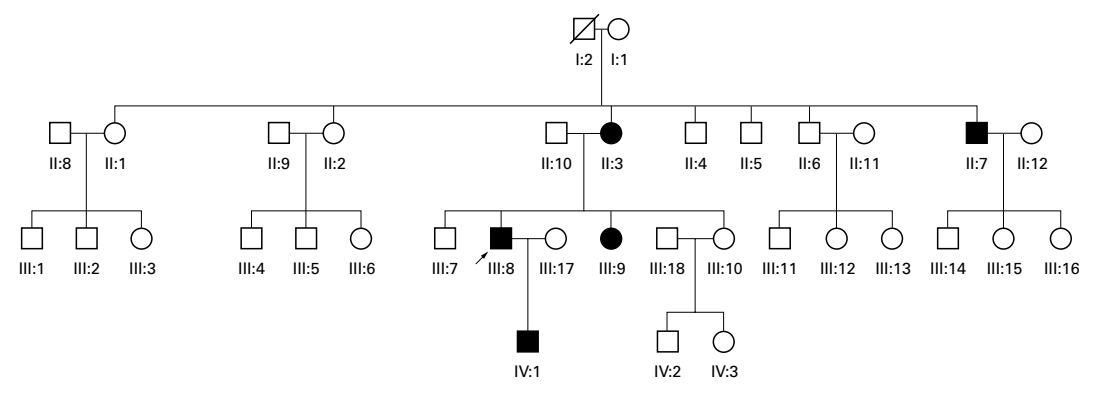

